\title{
BMJ Open Bariatric surgery and LDL cholesterol (BASALTO) trial study protocol: randomised controlled study evaluating the effect of gastric bypass versus sleeve gastrectomy on high LDL cholesterol
}

\author{
David Benaiges (1) , ${ }^{1,2,3,4}$ Albert Goday, ${ }^{1,2,3,5}$ Juana A Flores-Le Roux, ${ }^{1,2,3}$ \\ Montserrat Fitó, ${ }^{3,5}$ Oscar Pozo, ${ }^{6}$ Jose Rodríguez-Morató, ${ }^{6}$ Carme Serra, ${ }^{1}$ \\ Manuel Pera, ${ }^{7}$ Gemma Llauradó, ${ }^{1,2,3,4}$ Elisenda Climent, ${ }^{1,2,3}$ Olga Castañer, ${ }^{3,5}$ \\ Jose M Ramon, ${ }^{7}$ Juan Pedro-Botet ${ }^{1,2,3}$
}

To cite: Benaiges D, Goday A, Flores-Le Roux JA, et al. Bariatric surgery and LDL cholesterol (BASALTO) trial study protocol: randomised controlled study evaluating the effect of gastric bypass versus sleeve gastrectomy on high LDL cholesterol. BMJ Open 2020;10:e037712. doi:10.1136/ bmjopen-2020-037712

- Prepublication history for this paper is available online. To view these files, please visit the journal online (http://dx.doi org/10.1136/bmjopen-2020037712).

Received 13 February 2020 Revised 16 June 2020 Accepted 06 July 2020

Check for updates

(C) Author(s) (or their employer(s)) 2020. Re-use permitted under CC BY-NC. No commercial re-use. See rights and permissions. Published by BMJ.

For numbered affiliations see end of article.

Correspondence to

Dr David Benaiges;

96002@parcdesalutmar.cat

\section{ABSTRACT}

Introduction Observational studies have shown gastric bypass to be superior to sleeve gastrectomy in terms of low-density lipoprotein (LDL) cholesterol improvement. If these results are confirmed in randomised controlled trials, presurgical LDL cholesterol status could be a relevant factor in surgical procedure election. Furthermore, it is also necessary to establish the mechanisms by which LDL cholesterol improves after surgery and whether qualitative and quantitative changes occur in the different lipoprotein subclasses. The first objective is to ascertain whether high LDL cholesterol levels before surgery can be considered an additional factor when selecting the most appropriate surgical procedure for each patient (gastric bypass or sleeve gastrectomy). Hence, the 1-year remission rates of high LDL cholesterol after gastric bypass and sleeve gastrectomy in patients with morbid obesity will be compared. Secondary objectives were (1) to compare changes in other lipoproteins and LDL composition and (2) to study the pathophysiologic mechanisms related to LDL cholesterol remission.

Methods and analysis A randomised clinical trial, with intention-to-treat analysis, will be conducted to compare LDL cholesterol remission between gastric bypass and sleeve gastrectomy, with a 12-month follow-up. Inclusion criteria will be patients between 18 and 60 years of age with body mass index $\geq 40 \mathrm{~kg} / \mathrm{m}^{2}$ or $\geq 35$ $\mathrm{kg} / \mathrm{m}^{2}$ with significant obesity-related comorbidity and high LDL cholesterol levels. Patients will be evaluated preoperatively and at 3, 6 and 12 months after bariatric surgery. Examinations will include routine blood chemistry, anthropometric measurements, food intake recall, physical activity questionnaires and serum samples for lipidomic and lipoprotein characterisation.

Ethics and dissemination Ethics approval has been granted by the Parc de Salut Mar Ethics Committee (2019/8471/l). The study and its conclusions regarding the primary and secondary objectives will be presented as manuscripts submitted for peer-reviewed journal publication.

Trial registration number NCT03975478.
Strengths and limitations of this study

- This is the first randomised trial focused on bariatric surgery and low-density lipoprotein (LDL) cholesterol.

- The superiority of gastric bypass on LDL cholesterol reduction 1 year after the surgery needs to be confirmed.

- Baseline LDL cholesterol could be crucial in the surgical technique election between gastric bypass and sleeve gastrectomy.

- Moreover, this study wants to go a step further investigating pathophysiologic mechanisms related to LDL cholesterol remission.

- Patients with LDL cholesterol >190 mg/dL, familial hypercholesterolaemia or are in secondary prevention will not be included in the present trial.

\section{INTRODUCTION}

Obesity is associated with higher mortality, being related, at least in part, to a higher prevalence of cardiovascular risk factors such as type 2 diabetes, hypertension and hypercholesterolaemia. ${ }^{2}$ The characteristic dyslipidaemia of obesity involves different qualitative and quantitative lipoprotein alterations and contributes to the high cardiovascular risk in this population. ${ }^{3}$

Given the lack of effective medical therapeutic strategies, the demand for bariatric surgery (BS) has been growing worldwide over the last decade. Laparoscopic sleeve gastrectomy (LSG) and laparoscopic Rouxen-Y gastric bypass (LRYGB) are two of the most frequent procedures currently used for BS. ${ }^{4}$ In this context, the pros and cons of both techniques are crucial in clinical decision-making for the most appropriate 
BS procedure. ${ }^{5}$ In this respect, different observational studies reported the superiority of LRYGB in terms of low-density lipoprotein (LDL) cholesterol improvement, with no differences in other lipoproteins. Furthermore, a recent meta-analysis conducted by our group found hypercholesterolaemia remission to be more frequent 1 year after LRYGB than LSG (RR: 1.43, 95\% CI: 1.27 to 1.61), and, the drop in LDL cholesterol levels was greater after LRYGB than after LSG (mean difference: $19.29 \mathrm{mg} / \mathrm{dL}, 95 \%$ CI: 11.93 to 26.64$).{ }^{6}$ Concurring with these results, the SM-BOSS and SLEVEPASS trials (two randomised trials between LRYGB and LSG with a 5-year follow-up) reported lower LDL cholesterol levels with LRYGB but without the net LDL cholesterol decrease or the LDL cholesterol remission rate. ${ }^{78}$

Scientific evidence provided by randomised controlled trials (RCTs) on the metabolic effects of BS is scant. Moreover most RCT comparing LRYGB and LSG focused mainly on weight $\operatorname{loss}^{78}$ or type 2 diabetes remission, ${ }^{9} 10$ obviating the remaining comorbidities. In this respect, high scientific evidence studies in the field of BS focused on LDL cholesterol are lacking and could be crucial in surgical technique election.

\section{METHODS AND ANALYSIS \\ Primary objective}

The general aim is to ascertain whether high LDL cholesterol levels before surgery can be considered an additional factor when selecting the most appropriate surgical procedure for each patient (LRYGB or LSG). The main objective is to compare LDL cholesterol remission 1 year after LRYGB and LSG.

\section{Secondary objectives}

The secondary aims are (1) to compare the evolution of other lipoproteins (high-density lipoprotein (HDL) cholesterol, triglycerides and lipoprotein(a)) and LDL composition and (2) to study the mechanisms involved in the different LDL cholesterol remission rates after LRYGB and LSG.

\section{Patient and public involvement}

Neither patients nor the public are involved in the conception, design or conduct of the study.

\section{Study design}

A phase 3, single-centre, randomised clinical trial with an intention-to-treat analysis will be conducted to compare LDL cholesterol remission after LRYGB and LSG in patients with severe obesity and high LDL cholesterol levels. The follow-up period for the primary end-point will be 12 months, but patients will also be scheduled for a 5-year extension study.

\section{Inclusion criteria}

1. Body mass index (BMI) $\geq 40 \mathrm{~kg} / \mathrm{m}^{2}$ or $\geq 35 \mathrm{~kg} / \mathrm{m}^{2}$ with significant obesity-related comorbidities.

2. Age $18-60$ years.
3. Previous successfully instituted and supervised, but failed adequate diet and exercise programme.

4. Elevated LDL cholesterol defined as LDL cholesterol concentration $>130 \mathrm{mg} / \mathrm{dL}$ or treatment with cholesterol-lowering drugs.

\section{Exclusion criteria}

1. BMI $>60 \mathrm{~kg} / \mathrm{m}^{2}$.

2. Previous BS.

3. Exclusion criteria for BS.

i. Significant psychiatric disorder.

ii. Severe eating disorder, active alcohol or substance abuse.

iii. Contraindications for major abdominal surgery.

iv. Active gastric ulcer disease.

v. Severe liver disease.

vi. Pregnancy or breastfeeding.

4. Cases in which LSG or LRYGB are preferred.

i. Severe symptomatic gastro-oesophageal reflux disease despite medication.

ii. Large hiatal hernia.

iii. Expected presence of dense adhesions at small bowel level.

iv. Need for endoscopic follow-up of the duodenum, history of inflammatory bowel disease.

v. History of kidney transplantation in which drug malabsorption can be caused by a LRYGB.

5. Cholesterol-lowering drugs will be withdrawn immediately after surgery to assess LDL cholesterol remission during follow-up. Cases in which perioperative statin cannot be withdrawn will be excluded ${ }^{11}$ :

i. Established cardiovascular disease or subclinical cardiovascular disease (atheromatous plaque detection on carotid ultrasonography) in which LDL cholesterol objectives are more aggressive or statins can be prescribed regardless of LDL cholesterol levels.

ii. LDL cholesterol $>190 \mathrm{mg} / \mathrm{dL}$ or history of familial hypercholesterolaemia.

\section{Blinding}

There will be no blinding with regard to the type of surgery: patients as well as physicians and dietitians assessing follow-up data will be informed of the procedure performed. The researcher responsible for statistical analysis will be blinded to group allocation until data analysis is completed.

\section{Recruitment}

Participants will be recruited from the BS programme at the Hospital del Mar, Barcelona. Patients who meet the NIH criteria for BS (BMI $\geq 40 \mathrm{~kg} / \mathrm{m}^{2}$ or $\geq 35 \mathrm{~kg} / \mathrm{m}^{2}$ with significant obesity-related comorbidities) ${ }^{12}$ and who express the desire to undergo BS will be referred to the BS unit from primary care units. After inclusion in the BS programmes, patients will follow a standard nutritional intervention that includes 6-monthly group sessions focused on achieving changes in dietary habits and hence 
facilitating adaptation after surgery. After this group intervention, patients will be evaluated with routine blood chemistry by an endocrinologist. If they meet the inclusion criteria for the study and agree to participate, they will be scheduled for the inclusion visit.

\section{Inclusion visit}

An endocrinologist who will review the inclusion and exclusion criteria will evaluate the subjects. An ECG and supra-aortic trunk ultrasound will be performed. If established cardiovascular disease or atheromatous plaques are detected on ultrasonography, patients will not be included in the study. The proposal to participate in the study will be explained once again, doubts will be resolved and informed consent for the study will be signed. The patient will be given time to reconsider whether or not participate or to return a second time.

\section{Preoperative management}

Patients will follow the routine follow-up of those included in the Hospital del Mar BS programme from the time of study inclusion until the time of surgery. Different examinations will be performed (abdominal ultrasound, functional respiratory tests and gastroscopy) and results evaluated by a multidisciplinary team including dietitian, surgeon and endocrinologist. Ten days prior to surgery, a very low-calorie diet will be prescribed to achieve additional weight loss. In order to avoid bias that may arise due to an open study, a standardised protocol will be followed for the two groups regarding dietary recommendations and physical activity as well as the initiation of lipid-lowering medication after the intervention.

Management of cholesterol-lowering treatment will be based on the clinical practice guidelines of the Institut Català de la Salut. ${ }^{13}$ Cholesterol-lowering treatment will be started in primary prevention in adults with a 10 year estimated cardiovascular risk $>10 \%$ using REGICOR tables. ${ }^{14}$ Similarly, cholesterol-lowering treatment will be withdrawn in patients with a 10 year cardiovascular risk $<10 \%$ and with no previous LDL cholesterol determinations $>190 \mathrm{mg} / \mathrm{dL}$.

Two months before BS, a preoperative visit and tests will be carried out in patients included in the study. These will comprise routine blood chemistry, anthropometric measurements (weight, height and abdominal circumference), assessment of current lipid-lowering treatment, quantification of comorbidities other than high LDL cholesterol, food intake measured by 3-day food registry, physical activity measured by a specific questionnaire, serum bank collection for lipidomic determinations and lipoprotein characterisation, and faecal sample collection for gut microbiota examination.

\section{Randomisation}

The randomisation list will be electronically generated through specific software. Randomisation will be made before the preoperative visit using a sealed envelope, and the type of operation drawn will be explained again in detail to the subject.

\section{Surgical procedures}

The LRYGB technique consists of a $150 \mathrm{~cm}$ antecolic Roux limb with a $25 \mathrm{~mm}$ circular pouch-jejunostomy and the exclusion of $50 \mathrm{~cm}$ of the proximal jejunum. In LSG, longitudinal resection of the stomach from the angle of His to approximately $5 \mathrm{~cm}$ proximal to the pylorus is performed using a 35 French bougie inserted along the lesser curvature. The same team of surgeons will perform all operations.

\section{Postoperative visits}

Patients will be evaluated by the multidisciplinary team at 3,6 and 12 months after BS. Anthropometry, blood pressure, smoking status, routine blood chemistry, food intake registry, exercise questionnaire and serum bank will be collected at each visit. Additionally, faecal samples for gut microbiota examinations will be collected.

Statins and other lipid-lowering drugs will be withdrawn during hospital admission for the surgical procedure. Postoperatively, LDL cholesterol status will be evaluated at each visit:

1. LDL cholesterol $<130 \mathrm{mg} / \mathrm{dL}$ : cholesterol-lowering drugs will not be initiated and patients will be considered in remission.

2. LDL cholesterol 130-190 mg/dL: cholesterol-lowering drugs will be initiated in patients with persistent estimated cardiovascular risk $>10 \%$ using REGICOR charts. $^{14}$

3. LDL cholesterol >190 mg/dL: cholesterol-lowering drugs will be initiated.

Patients will also receive nutritional support in the immediate postoperative period (at 2, 4 and 8 weeks) with the aim of progressing from a liquid to a soft solid diet. Thereafter, appointments will be held at 6 and 12 months to detect and manage possible dietary intolerances. At 1 year postsurgery, the diet should be solid, varied and low calorie.

\section{Outcome definition}

\section{Lipid disturbance evaluation}

1. LDL cholesterol remission: postoperative LDL cholesterol $<130 \mathrm{mg} / \mathrm{dL}$ without cholesterol-lowering drugs.

2. LDL cholesterol improvement:

i. Patients without preoperative cholesterol-lowering drugs: decrease $\geq 20 \%$ in LDL cholesterol concentration without cholesterol-lowering drugs.

ii. Patients with preoperative cholesterol-lowering drugs: medication withdrawn and LDL cholesterol $>130 \mathrm{mg} / \mathrm{dL}$ or decrease $\geq 20 \%$ in LDL cholesterol concentration without medication withdrawn.

3. Hypertriglyceridaemia remission: triglycerides $<150$ $\mathrm{mg} / \mathrm{dL}$ without fibrates.

4. Low HDL cholesterol remission: HDL cholesterol $>50$ $\mathrm{mg} / \mathrm{dL}$ in women or $>40 \mathrm{mg} / \mathrm{dL}$ in men. 


\section{Other cardiovascular outcomes}

1. Type 2 diabetes complete remission: glycated haemoglobin (HbA1c) value $<6.0 \%$ and fasting glucose level $<100 \mathrm{mg} / \mathrm{dL}$ without diabetes medications. ${ }^{15}$

2. The 10-year risk of cardiovascular disease will be estimated using the REGICOR adaptation of the Framingham Risk Score for the Mediterranean population. ${ }^{14}$ Risk factors included in the calculation are age, gender, total cholesterol levels, HDL cholesterol levels, diastolic and systolic blood pressures, type 2 diabetes and smoking status.

\section{Anthropometric parameters}

Height will be measured at the inclusion visit with a high precision measuring rod. Weight and blood pressure will be measured at each visit. Patients are measured in lightweight clothing. BMI will be calculated as weight in kilograms divided by the square of height in metres. Weight loss will be measured as the percentage of excess BMI loss $(100 \times$ [baseline BMI-follow-up BMI] / [baseline BMI-25]) and percentage of excess weight loss (initial weight-follow-up weight) / (initial weight-ideal weight for BMI 25) $\times 100 \%$.

\section{Laboratory methods}

Routine blood chemistry performed after a 12 hours overnight fast will be analysed at our reference laboratory. Complete lipid profile, glucose, HbA1c, kidney and liver function will be determined. Plasma samples will be collected and frozen at $-80^{\circ} \mathrm{C}$. Lipoprotein characterisation and lipidomic determinations will be carried out in all patients when they complete follow-up.

Total cholesterol, LDL cholesterol, HDL cholesterol and triglycerides will be determined using enzymatic methods (Roche Cobas 8000 c701, Roche Diagnostics $\mathrm{GmbH}$, Germany) and lipoprotein(a) by immunoturbidimetric determination (Roche Cobas 8000 c502, Roche Diagnostics GmbH, Germany).

Targeted lipidomic approaches developed at the Clinical Metabolomics Platform at Hospital del Mar Medical Research Institute will be applied to provide new insights into the mechanism(s) responsible for the BS-induced lowering effects on LDL cholesterol. The lipid families/ pathways planned to be targeted are as follows: (1) precursors and metabolites of cholesterol, (2) bile acids, (3) oxysterols, (4) cholesteryl esters, (5) steroid hormones and metabolites, (6) ceramides, (7) diacylglycerols and (8) lysophosphatidylcholines. Based on the Platform's experience, methods based on liquid or gas chromatography coupled with mass spectrometry (LC-MS/MS or- GC-MS) will be applied to detect the targeted lipid molecules. Serum/plasma and/or stool samples will be divided into aliquots for the different lipidomic methods. After addition of the appropriate labelled internal standards, samples will be extracted using solid-phase or liquidliquid process and/or derivatised. Analytes contained in the final extract will be determined by LC-MS/MS or GC-MS using a specific set-up for each selected pathway (column, mobile phase) and by the acquisition of specific selected reaction monitoring transitions (in LC-MS/ MS) or using selected ion monitoring (in GC-MS) for each analyte. Quantification will normally be performed either by external calibration or relative response factor employing the ratio between the analyte and the labelled internal standard as a response. In order to assure the between-batch reproducibility of the measurements, two quality control samples will be analysed in every batch.

Characterisation of the different lipoproteins will be made by nuclear magnetic resonance using an advanced lipoprotein test (LIPOSCALE TEST). This determines cholesterol and triglyceride content of the main lipoprotein fractions (Very Low Density Liporpotein (VLDL), Intermediate Density Lipoprotein (IDL), LDL and HDL) in plasma and blood serum. It also analyses the particle concentration (large, medium and small) of each main fraction (VLDL, LDL and HDL) and, finally, the size of the main lipoprotein fractions (VLDL, LDL and HDL). ${ }^{16}$

\section{Microbiota}

Fresh faeces will be collected and immediately frozen and stored at $-80^{\circ} \mathrm{C}$ in our centre's freezers until processed. The microbial composition will be processed using illumina sequencing technique. We will perform microbial DNA genomic and total RNA extraction, sequencing of $16 \mathrm{~S}$ rDNA and 16S rRNA, metagenomic sequencing; purification, amplification and sequencing of mRNA; metagenomic and metatranscriptomic analyses, protein extraction together with separation, identification and processing of data.

\section{Adipose tissue biopsy}

During surgery, 2-5 g visceral adipose tissue and subcutaneous adipose tissue samples will be removed. Visceral adipose tissue will be obtained from perigastric adipose tissue, and subcutaneous adipose tissue from the site of the surgical incision. The adipose tissue samples will be processed immediately, cut into small pieces, washed with Phosphate Buffered Saline (PBS) and frozen at $-80^{\circ} \mathrm{C}$ until further analysis. The subsequent analysis of gene expression will be performed by quantitative polymerase chain reaction (qPCR) and western blot techniques.

\section{Food intake and physical activity}

All subjects will have to fill in a dietary record for three consecutive days from Sunday to Tuesday. Nutrient intakes will be calculated with the DIET ANALYSIS NUTRITIONIST IV software (N Squared Computing, San Bruno, California, USA).

Physical activity will be measured using the REGICOR Short Physical Questionnaire. ${ }^{17}$ This questionnaire covers all four dimensions of physical activity: type, frequency, duration and intensity and lists six types of activity: walking, brisk walking, walking on trails/hiking, gardening, climbing stairs and sport. To complete the questionnaire, trained personnel will ask participants the 
number of days per month and average minutes per day they performed the activity.

\section{Sample size}

Assuming LDL cholesterol remission rates to be $70 \%$ in the LRYGB group and 20\% in the LSG, a minimum of 18 patients per arm will provide $80 \%$ power to detect a difference between groups using a two-sided $\alpha$ of 0.05 . These estimates were derived from our previous published data. ${ }^{18}$ A drop-out rate of $20 \%$ has been anticipated.

\section{Data analysis}

All data will be collected in a central database according to a standardised protocol. Data will be expressed as mean \pm SD for continuous variables after a normal distribution, as median with IQR for continuous variables with a non-normal distribution and as percentages and frequencies for categorical variables. Normality of the models will be evaluated visually and by the Kolmogorov-Smirnov test. For skewed variables, a logarithmic transformation will be used to achieve normality. Student's t-test will be performed to assess differences between two means. $\chi^{2}$ or Fisher's exact tests will be used to assess the degree of association among categorical variables (including the primary outcome: LDL cholesterol remission at 1 year). Analysis of variance models will be used to study the evolution of continuous variables in each group and analyse differences between groups at each time point from baseline. The effect of BS on lipidome will be analysed with mixed generalised linear models for repeated measurements, adjusting for covariates such as weight, BMI and physical activity, among others. A two-sided $\mathrm{p}$ value $<0.05$ will be considered statistically significant.

\section{ETHICS AND DISSEMINATION}

The study will be conducted in accordance with the basic principles of protection of rights and dignity of the human being, as stated in the Helsinki Declaration and with current regulations. Law 14/2007 of Biomedical Research (LIB) will be adhered to the studies with biologic samples. All the information obtained will be treated confidentially in compliance with the personal data protection legislation (Ley Orgánica 3/2018, de 5 de diciembre, de Protección de Datos Personales y garantía de los derechos digitales). Participants will sign their informed consent to participate in the study. Ethics approval has been granted by the Parc de Salut Mar Ethics Committee (2019/8471/I). The study and its conclusions regarding the primary and secondary objectives will be presented as manuscripts submitted for peer-reviewed journal publication.

\section{DISCUSSION}

LRYGB has been considered the gold standard and most used BS technique for several years owing to its superior risk-benefit balance, better results than restrictive techniques and fewer complications than purely malabsorptive surgeries. ${ }^{19}$ Nevertheless, the use of LSG has increased in the last 10 years, thanks to its technical simplicity and similar short-term results than LRYGB in terms of weight loss and comorbidity remission such as type 2 diabetes and hypertension. ${ }^{7-9}$ These advantages placed LSG for the first time as the most widely used BS technique worldwide in 2014, overtaking LRYGB. ${ }^{4}$ In this context, there is intense debate in the scientific community as to whether LSG can replace LRYGB as the new gold standard. Following publication of the SLEEVEPASS and SM-BOSS trials, Arterburn et a $\tilde{l}^{\tilde{y}}$ published an editorial in the JAMA where they considered that switching from LRYGB to LSG has not been a misadventure and that either technique is a great decision. Moreover, they highlighted that five different points need to be considered in the difficult choice between the two: (1) the election has to be decided jointly between the patient and medical team, (2) there are no differences in weight loss, (3) the presence of type 2 diabetes does not render LRYGB preferable to LSG, (4) LRYGB is preferred in patients with gastrooesophageal reflux disease and (5) there are no differences in the rate of postsurgical reoperation for different reasons (gastro-oesophageal reflux with LSG and small bowel obstruction or internal hernia with LRYGB). Nevertheless, if the BASALTO trial confirms the superiority of LRYGB over LSG in terms of LDL cholesterol remission, we believe this should be the sixth point to be taken into account in the surgical procedure decision.

This trial will attempt to go one step further to study the pathophysiologic mechanism(s) involved in LDL changes after both techniques. As reported by our group in previous studies, LDL cholesterol improvement is greater in patients with higher baseline LDL cholesterol levels and is not related to postoperative weight loss. The predominant mechanism involved in LDL cholesterol after LRYGB appears to be malabsorption. In this respect, Pihlajamäki et $a l^{20}$ found a reduction in cholesterol absorption markers only after LRYGB, an effect not previously reported following a restrictive technique (gastric banding). Moreover, other authors suggest that the LDL cholesterol decrease could be related to bile acid metabolism. ${ }^{21}$ Nevertheless, if the only mechanism is malabsorption, we cannot explain why approximately one in every five patients operated on with LSG can achieve LDL cholesterol remission. Analysis of the lipidome in BASALTO study subjects will permit us to identify the changes in plasma concentrations of different lipid compounds due to surgical interventions and determine which metabolic pathways would be modulated with each technique to later correlate this information with the alterations observed in LDL cholesterol. Thus, the targeted lipidomic analysis of the present study will be essential to obtain an overall view that affords understanding of the changes in LDL cholesterol with each technique.

On the other hand, that the atherogenic profile of LDL particles may vary depending on their size and 
composition is well known. Thus, the presence of small and dense LDL particles has been associated with obesity, diabetes and increased cardiovascular risk. Evidence of the effects of BS on the size and composition of LDL is limited. In this regard, Kjellmo et al reported that biliopancreatic diversion and LRYGB reduce the number of large LDL particles but have no effect on small and dense LDL. ${ }^{22}$ To our knowledge, no studies have been conducted on the effects of LSG on the size and composition of LDL.

This study has certain limitations. Since patients with LDL cholesterol $>190 \mathrm{mg} / \mathrm{dL}$, familial hypercholesterolaemia or those on secondary prevention will not be included in the present trial, the results will not be able to be extrapolated to the overall population with severe obesity and high LDL cholesterol concentrations. However, it must be highlighted that the number of patients on secondary prevention who undergo BS is low. In this respect, only $2.2 \%$ patients included in the Swedish Obese Subjects study presented myocardial infarction or stroke previous to surgery. ${ }^{23}$ Moreover, in patients receiving treatment with cholesterol-lowering drugs before surgery, it will not be possible to assess the absolute changes in LDL cholesterol concentration, and changes in lipoprotein size and composition should be interpreted with caution.

\section{TRIAL STATUS}

This study obtained the Ethics Committee approval in March 2019 and was registered at clinicaltrials.gov in June 2019. Enrolment in the trial began in July 2019 and 11 of the 36 required subjects have been recruited to date. A Spanish National health System Grant was recently adjudicated (PI19/00006). This study is sponsored by a Public Health Hospital (Parc de Salut Mar).

Sixty BS procedures are currently performed per year in our institution. In a recent study conducted at our centre, $47 \%$ of the operated patients presented high LDL cholesterol levels. ${ }^{18}$ Taking into account that the estimated sample size is 36 , recruitment can be expected to be completed in 1.5 years. If the preoperative and postoperative times are considered, follow-up of all the included patients should be completed in 3-3.5 years. Finally, 6 months will be required to analyse the data and publish the results obtained in scientific journals.

\footnotetext{
Author affiliations

${ }^{1}$ Endocrinology and Nutrition, Hospital del Mar, Barcelona, Spain

${ }^{2}$ Department of Medicine, Universitat Autònoma de Barcelona, Barcelona, Spain ${ }^{3}$ Institut Hospital del Mar d'Investigacions Mèdiques (IMIM), Barcelona, Spain

${ }^{4}$ Consorci Sanitari de I'Alt Penedès Garraf, Vilafranca del Penedès, Spain

${ }^{5}$ CiberOBN. Instituto de Salud Carlos III, Madrid, Spain

${ }^{6}$ Integrative Pharmacology and Systems Neuroscience Research Group, Institut

Hospital del Mar d'Investigacions Mèdiques, Barcelona, Spain

${ }^{7}$ Department of General Surgery, Hospital del Mar, Barcelona, Spain
}

Acknowledgements We thank Miss Christine 0'Hara for review of the English version of the manuscript.

Contributors DB performed the study conceptualisation, data curation, funding acquisition, methodology, investigation, project administration, supervision and creating the original draft. AG helped in conceptualisation, project administration, supervision, review and editing of draft. JF-LR performed the project administration and supervision. MF helped in funding acquisition, methodology, project administration and supervision. OP helped in conceptualisation, data curation, funding acquisition, methodology and investigation. JR-M performed study conceptualisation, data curation, funding acquisition, methodology and investigation. CS helped in investigation. GL helped in data curation and methodology. EC helped in Investigation. OC performed funding acquisition, data curation, methodology and project administration. JMR helped in conceptualisation, methodology, investigation, project administration and supervision. JP-B performed conceptualisation, methodology, funding acquisition, project administration, supervision, review and editing of draft.

Funding This work was supported by (Spanish National Health System Grant) grant number [P119/00006].

Competing interests None declared.

Patient and public involvement Patients and/or the public were not involved in the design, or conduct, or reporting, or dissemination plans of this research.

Patient consent for publication Not required.

Provenance and peer review Not commissioned; externally peer reviewed.

Open access This is an open access article distributed in accordance with the Creative Commons Attribution Non Commercial (CC BY-NC 4.0) license, which permits others to distribute, remix, adapt, build upon this work non-commercially, and license their derivative works on different terms, provided the original work is properly cited, appropriate credit is given, any changes made indicated, and the use is non-commercial. See: http://creativecommons.org/licenses/by-nc/4.0/.

ORCID iD

David Benaiges http://orcid.org/0000-0001-5411-364X

\section{REFERENCES}

1 Allison DB, Fontaine KR, Manson JE, et al. Annual deaths attributable to obesity in the United States. JAMA 1999;282:1530-8.

2 Must A, Spadano J, Coakley EH, et al. The disease burden associated with overweight and obesity. JAMA 1999;282:1523-9.

3 Grundy SM. Obesity, metabolic syndrome, and cardiovascular disease. J Clin Endocrinol Metab 2004;89:2595-600.

4 Angrisani L, Santonicola A, lovino P, et al. Bariatric surgery and endoluminal procedures: IFSO worldwide survey 2014. Obes Surg 2017;27:2279-89.

5 Arterburn D, Gupta A. Comparing the outcomes of sleeve gastrectomy and Roux-en-Y gastric bypass for severe obesity. JAMA 2018;319:235.

6 Climent E, Benaiges D, Pedro-Botet J, et al. Laparoscopic Rouxen-Y gastric bypass vs. laparoscopic sleeve gastrectomy for morbid obesity: a systematic review and meta-analysis of lipid effects at one year postsurgery. Minerva Endocrinol 2018;43:87-100.

7 Salminen P, Helmiö M, Ovaska J, et al. Effect of laparoscopic sleeve gastrectomy vs laparoscopic Roux-en-Y gastric bypass on weight loss at 5 years among patients with morbid obesity: the sleevepass randomized clinical trial. JAMA 2018;319:241-54.

8 Peterli R, Wölnerhanssen BK, Peters T, et al. Effect of laparoscopic sleeve gastrectomy vs laparoscopic Roux-en-Y gastric bypass on weight loss in patients with morbid obesity: the SM-BOSS randomized clinical trial. JAMA 2018;319:255-65.

9 Schauer PR, Kashyap SR, Wolski K, et al. Bariatric surgery versus intensive medical therapy in obese patients with diabetes. $N$ Engl J Med 2012;366:1567-76.

10 Casajoana A, Pujol J, Garcia A, et al. Predictive value of gut peptides in T2D remission: randomized controlled trial comparing metabolic gastric bypass, sleeve gastrectomy and greater curvature plication. Obes Surg 2017;27:2235-45.

11 Grundy SM, Stone NJ, Bailey AL, et al. 2018 AHA/ACC/ AACVPR/AAPA/ABC/ACPM/ADA/AGS/APhA/ASPC/NLA/PCNA guideline on the management of blood cholesterol. Circulation 2018;139:e1082-143.

12 Rubino F, Kaplan LM, Schauer PR, et al. The diabetes surgery summit consensus conference. Ann Surg 2010;251:399-405. 
13 Ciurana Misol R, Franzi Sisó A, García Cerdán MR, et al. Guíes de práctica clínica. Colesterol I RISC coronari. General Catalunya Inst Catal la Salut 2009:1-131.

14 Marrugat J, D'Agostino R, Sullivan L, et al. An adaptation of the Framingham coronary heart disease risk function to European mediterranean areas. J Epidemiol Community Health 2003;57:634-8.

15 Buse JB, Caprio S, Cefalu WT, et al. How do we define cure of diabetes? Diabetes Care 2009;32:2133-5.

16 Mallol R, Amigó N, Rodríguez MA, et al. Liposcale: a novel advanced lipoprotein test based on 2D diffusion-ordered 1H NMR spectroscopy. J Lipid Res 2015;56:737-46.

17 Molina L, Sarmiento M, Peñafiel J, et al. Validation of the REGICOR short physical activity questionnaire for the adult population. PLOS One 2017;12:e0168148.

18 Climent E, Benaiges D, Flores-Le Roux JA, et al. Changes in the lipid profile 5 years after bariatric surgery: laparoscopic Roux-en-Y gastric bypass versus laparoscopic sleeve gastrectomy. Surg Obes Relat Dis 2018;14:1099-105.
19 Buchwald H, Avidor Y, Braunwald E, et al. Bariatric surgery: a systematic review and meta-analysis. JAMA 2004;292:1724-37.

20 Pihlajamäki J, Grönlund S, Simonen M, et al. Cholesterol absorption decreases after Roux-en-Y gastric bypass but not after gastric banding. Metabolism 2010;59:866-72.

21 Ferrannini E, Camastra S, Astiarraga B, et al. Increased bile acid synthesis and deconjugation after biliopancreatic diversion. Diabetes 2015;64:3377-85.

22 Kjellmo CA, Karlsson H, Nestvold TK, et al. Bariatric surgery improves lipoprotein profile in morbidly obese patients by reducing LDL cholesterol, apoB, and SAA/PON1 ratio, increasing HDL cholesterol, but has no effect on cholesterol efflux capacity. J Clin Lipidol 2018;12:193-202.

23 Sjöström L, Lindroos A-K, Peltonen M, et al. Lifestyle, diabetes, and cardiovascular risk factors 10 years after bariatric surgery. $N$ Engl $J$ Med 2004;351:2683-93. 